\title{
An Implicit Numerical Model Using Split Flux for 1-D Unsteady Flows With Shocks
}

\author{
By Akhilesh Kumar JHA ${ }^{*}$, Juichiro AKIYAMA ${ }^{* *}$, and Masaru URA ***
}

\begin{abstract}
An implicit model based on splitting of flux and automatic switching of space differences is presented to solve one-dimensional unsteady flows with shocks. The scheme switches from central to upwind if the flow changes from subcritical to supercritical and vise versa. The model described here is simple and straightforward to formulate and program. This model is specially suited to simulate shock propagation and flow situations where both, subcritical and supercritical flows, are present. Detail of the model and illustrative examples are also discussed.
\end{abstract}

Keywords:Unsteady flow, Shocks, Finite difference, Implicit, Splitflux

\section{INTRODUCTION}

The unsteady free surface flows generated by sudden and complete opening or closure of a channel or by removal of dam from the entire width of the channel can be simulated by one-dimensional model. There exist many explicit and implicit models capable of simulating one-dimensional unsteady free surface flows and they are extensively covered in the literature $1,2,3)$. The problem of simulating unsteady flows becomes quite involved if the bores or shocks are formed leading to the discontinuous solution. There are two commonly used approaches to handle such situations. The first approach, known as shock-fitting 4,5 ), isolates the bore and computes its propagation for one time step independently of the computation in the two adjacent continuous regions. The second approach, known as shock capturing, does not treat the shock as an internal boundary and the solution is obtained by integrating the governing equations in conservation form.

In order to correctly handle the bore and the flow situations where both supercritical and subcritical flows are present simultaneously or in sequence it is necessary to properly implement the directional property of signal propagation inherent in the hyperbolic nature of the governing equations. In general, schemes based on central space difference are most likely to fail in such situations because part of the information is transmitted from the wrong direction. Method of characteristics ${ }^{6,7)}$ correctly applies the concept of signal propagation but fixed grid finite difference schemes are favoured for computer applications. Besides, extension of the method of characteristics to two-dimensions becomes too cumbersome to handle. Some finite difference schemes have also incorporated directional property of signal propagation through various techniques, often leading to complicated algorithms. For example, spatial differencing may be switched through a control function in Abbott and Ionescu scheme ${ }^{8)}$ whereas, in two-dimensional Leap-Frog scheme differencing based on flow direction was incorporated ${ }^{9}$ ).

The split flux algorithm can handle appropriately the directional property on signal propagation and can be combined with a suitable finite difference scheme. In this algorithm the flux vectors are split into positive and negative components based on signs of eigenvalus of jacobian of flux matrices. The upwind differencing is used for the flow vectors associated with positive component of flux vector and downwind differencing is used for the flow vectors associated with negative component of flux vector. Explicit schemes ${ }^{10,11)}$ based on split flux use two and three step predictor-corrector algorithm involving seven and five grid points respectively. Beam and Warming ${ }^{12}$ ) scheme, which is an implicit scheme developed for fluid dynamics, also uses split flux technique but its development is rather complicated.

\footnotetext{
* M.Eng., Graduate Student, Dept. of Civil Engg., Kyushu Institute of Technology

(Tobata, Kitakyushu, 804)

** Member of JSCE, Ph.D., Associate Professor

*** Member of JSCE, Dr. Engg., Professor
} 
In this paper, a simple and easy implicit numerical model based on splitting of flux and switching technique is newly developed to simulate one-dimensional unsteady free surface flows with shocks. Treatment of the numerical boundaries are also discussed. For the purpose of demonstrating applicability and validity of the model, results of the present model are compared with analytical solution for horizontal, frictionless channel and/or with results of previous numerical studies.

\section{GOVERNING EQUATIONS}

The governing equations based on the conservation of mass and momentum for one-dimensional unsteady free-surface flow may be expressed as

$$
\frac{\partial \mathbf{U}}{\partial t}+\frac{\partial \mathbf{E}}{\partial \mathbf{x}}+\mathbf{S}=0
$$

where,

$$
\mathbf{U}=\left[\begin{array}{c}
h \\
u h
\end{array}\right] \quad ; \quad \mathbf{E}=\left[\begin{array}{c}
u h \\
u^{2} \cdot h+\frac{1}{2} \cdot g h^{2}
\end{array}\right], \quad \text { and } \mathbf{S}=\left[\begin{array}{c}
0 \\
-g h\left(S_{o}-S_{f}\right)
\end{array}\right]
$$

where, $\mathrm{h}=$ flow depth, $\mathrm{u}=$ velocity, $\mathrm{g}=$ acceleration due to gravity; $\mathrm{S}_{\mathrm{o}}, \mathrm{S}_{\mathrm{f}}=$ bed slope and friction slope respectively. There are four assumptions basic to the governing equations given above: (1) Water is incompressible; (2) pressure is hydrostatic; (3) bottom slope of the channel is sufficiently small; and (4) geostrophic effects and wind stresses are negligible.

Eq. (1) can also be expressed in terms of Jacobian, $\mathbf{A}$ of $\mathbf{E}$ by noting the following

$$
\frac{\partial \mathbf{E}}{\partial \mathbf{x}}=\mathbf{A} \frac{\partial \mathbf{U}}{\partial \mathbf{x}} \quad \text { where, } \mathbf{A}=\left[\begin{array}{cc}
0 & 1 \\
-u^{2}+g h & 2 u
\end{array}\right]
$$

Therefore, the governing equations become;

$$
\frac{\partial \mathbf{U}}{\partial t}+\mathbf{A} \cdot \frac{\partial \mathbf{U}}{\partial \mathbf{x}}+\mathbf{S}=0
$$

Matrix $\mathbf{A}$ can be written in diagonalized form as

$$
\mathbf{A}=\frac{1}{2 c}\left[\begin{array}{cc}
(u+c) \lambda_{2}-(u-c) \lambda_{1} & \lambda_{1}-\lambda_{2} \\
(u-c)(u+c)\left(\lambda_{2}-\lambda_{1}\right) & (u+c) \lambda_{1}-(u-c) \lambda_{2}
\end{array}\right]
$$

where, $c=(g h)^{1 / 2}$ and $\lambda_{i}$ 's are eigenvalues of $\mathbf{A}$ giving the characteristic directions. The eigenvalues are given by

$$
\lambda_{1}=\mathrm{u}+\mathrm{c} \quad \text { and } \quad \lambda_{2}=\mathrm{u}-\mathrm{c}
$$

Now, matrix $\mathbf{A}$ can be split into two components, positive and negative, by testing sign of the eigenvalues. This is done as follows,

$$
\mathbf{A}=\mathbf{A}^{+}+\mathbf{A}^{-} \quad \text { where, } \lambda_{i}^{+}=\max \left(\lambda_{i}, 0\right) \quad \text {, and } \lambda_{i}^{-}=\lambda_{i}-\lambda_{i}^{+}
$$

The governing Eq.(3) can now be written in split flux form as

$$
\frac{\partial \mathbf{U}}{\partial t}+\mathbf{A}^{+} \cdot \frac{\partial \mathbf{U}}{\partial \mathbf{x}}+\mathbf{A}^{-} \cdot \frac{\partial \mathbf{U}}{\partial \mathrm{x}}+\mathbf{S}=0
$$

The reason for the above manipulations is that the derivative associated with positive component of matrix $\mathbf{A}$ can be approximated by backward space difference and that associated with negative component of matrix $\mathbf{A}$ by forward space difference. It may be noted that although Eq. (1) is in conservative form Eq. (7) no longer remains in conservative form. Euler equations of gas dynamics have the interesting property that

$$
\frac{\partial \mathbf{E}}{\partial \mathbf{x}}=\mathbf{A} \frac{\partial \mathbf{U}}{\partial \mathbf{x}}=\frac{\partial(\mathbf{A} \mathbf{U})}{\partial \mathbf{x}}
$$

because $\mathbf{E}$ is homogeneous function of degree one in $\mathbf{U}$. However, $\mathbf{E}$ in Eq. (1) is homogeneous function of degree two in $\mathbf{U}$ and, therefore, Eq. (8) is not valid. Consequently, conservative splitting of $\mathbf{E}$ is not possible in the prescnt casc. However, conservative splitting may be achieved for continuily equation because element of $\mathbf{E}$ corresponding to the continuity equation is homogeneous function of degree one in the corresponding $\mathbf{U}$. In the following text elements of matrix $\mathbf{A}$ will be refered to as, for example, Aij, referring to the element corresponding to row $\mathrm{i}$ and column $\mathrm{j}$ of matrix $\mathbf{A}$. Following this notation and writing continuity equation in conservation form, Eq.(3) may be expanded from its matrix form to give following equations. 
Continuity:

$$
\frac{\partial \mathrm{h}}{\partial \mathrm{t}}+\frac{\partial\left(\mathrm{A}_{11} \mathrm{~h}\right)}{\partial \mathrm{x}}+\frac{\partial\left(\mathrm{A}_{12} \mathrm{uh}\right)}{\partial \mathrm{x}}=0
$$

$\mathrm{X}$-momentum:

$$
\frac{\partial(\mathrm{uh})}{\partial \mathrm{t}}+\mathrm{A}_{21} \frac{\partial \mathrm{h}}{\partial \mathrm{x}}+\mathrm{A}_{22} \frac{\partial(\mathrm{uh})}{\partial \mathrm{x}}-\mathrm{gh}\left(\mathrm{S}_{\mathrm{o}}-\mathrm{S}_{\mathrm{f}}\right)=0
$$

The conservation form of continuity equation, i.e. Eq.(9), can be verified by simply substituting values of $A_{11}$ and $A_{12}$ in Eq.(9) and comparing with Eq.(1). Therefore, Eq.(9) and (10) can be written in split flux form as

Continuity:

$$
\frac{\partial h}{\partial t}+\frac{\partial\left(A_{11}^{+} h\right)}{\partial x}+\frac{\partial\left(A_{11}^{-} h\right)}{\partial x}+\frac{\partial\left(A_{12}^{+} u h\right)}{\partial x}+\frac{\partial\left(A_{12}^{-} u h\right)}{\partial x}=0
$$

X-momentum:

$$
\frac{\partial(u h)}{\partial t}+A_{21}^{+} \frac{\partial h}{\partial x}+A_{21}^{-} \frac{\partial h}{\partial x}+A_{22}^{+} \frac{\partial(u h)}{\partial x}+A_{22}^{-} \frac{\partial(u h)}{\partial x}-g h\left(S_{o}-S_{f}\right)=0
$$

where, + and - superscripts on $A_{i j}$ indicate positive and negative components of $A$ respectively. The friction slope in Eq.(12) is assumed to be given by Manning's formula. Thus,

$$
S_{f}=\frac{n^{2} \cdot u \cdot|u|}{h^{4 / 3}}
$$

where, $\mathrm{n}=$ Manning's roughness coefficients.

\section{FINITE DIFFERENCE SCHEME}

Writing Eq.(11) and (12) in finite difference form is rather straightforward. Time derivatives are approximated by a forward difference whereas space derivatives are replaced by either a backward or forward difference depending on whether they are associated with positive or negative components of $A_{i j}$ respectively. We make use of the following operators

$$
\Delta_{x} f_{i}=f_{i+1}-f_{i}, \nabla_{x} f_{i}=f_{i}-f_{i-1} \text { and } \nabla_{t} f_{i}=f_{i}^{t+1}-f_{i}^{t}
$$

and define $\alpha=\Delta x / \Delta t$, where, superscripts and subscripts in Eq.(14) stand for location in time and space respectively (Fig. 1). $\Delta \mathrm{x}$ is grid interval and $\Delta \mathrm{t}$ is the time step. The finite difference equation for Eq.(11) and (12) is respectively written as

$$
\begin{array}{r}
\nabla_{t} h_{i}+\alpha\left\{\nabla_{X}\left[\left(A_{11}^{+}\right)^{t} h^{t+1}\right]_{i}+\Delta_{X}\left[\left(A_{11}^{-}\right)^{t} h^{t+1}\right]_{i}+\nabla_{X}\left[\left(A_{12}^{+}\right)^{t}(u h)^{t+1}\right]_{i}+\Delta_{X}\left[\left(A_{12}^{-}\right)^{t}(u h)^{t+1}\right]_{i}\right\}=0 \\
\nabla_{t}(u h)_{i}+\alpha\left\{\left(A_{21}^{+}\right)_{i}^{t} \nabla_{X} h_{i}^{t+1}+\left(A_{21}^{-}\right)_{i}^{t} \Delta_{x} h_{i}^{t+1}+\left(A_{22}^{+}\right)_{i}^{t} \nabla_{X}(u h)_{i}^{t+1}+\left(A_{22}^{-}\right)_{i}^{t} \Delta_{x}(u h)_{i}^{t+1}-g h_{i}^{t+1} S_{o}\right\} \\
+\Delta t \cdot g(u h)_{i}^{t+1}\left[\frac{n^{2}|u|}{h^{4 / 3}}\right]_{i}^{t}=0 \ldots \ldots . .
\end{array}
$$

For subcritical flows, Eqs.(15) and (16) implement central space differencing with the appropriate weighting governed by eigenvalues given by Eq.(5). For supercritical flows, the scheme given by Eqs.(15) and (16) automatically switch to full upwind because negative components of $\mathbf{A}$ are removed by Eq.(6). Similar sets of finite difference equations can be written for all nodes along a channel. The resulting system of equation can be arranged in the form of a block tri-diagonal matrix with each block of size $(2 \mathrm{x}$ 2). This block tri-diagonal system can be solved by any suitable algorithm. In this study it has been solved by the algorithm given by S.R. Chakravarthy ${ }^{13)}$.

Boundary and initial conditions must be correctly specified and incorporated into the scheme to obtain

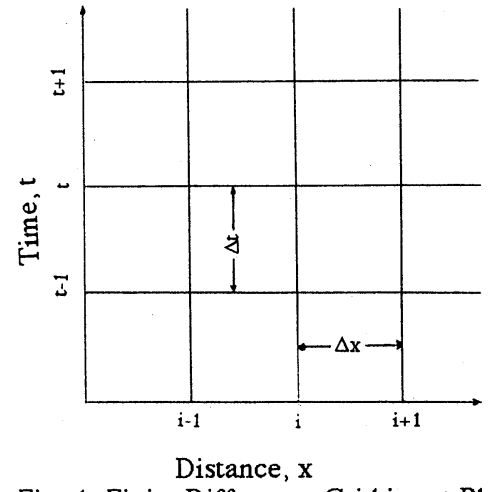

Fig. 1 Finite Difference Grid in $x-t$ Plane 
correct results. In the split flux algorithm boundary and initial conditions are easily incorporated. The compatibility equations valid along each characteristic are obtained and the appropriate one is replaced by the specified boundary condition. For example, in case of a closed upstream boundary, the characteristic coming from the wall may be replaced by the condition of zero mass flux through the wall i.e. (uh) $=0$ and the depth may be computed by the compatibility equation along the characteristic coming from inside the domain. Two types of boundary conditions are considered in this study, closed and open. A closed boundary is a surface of zero mass flux across it and can be represented by a solid wall. It is assumed that the solid wall has an orientation always parallel to the sides of square grid and the velocity component perpendicular to the wall is set to zero. In case of open boundaries various possible boundary conditions may be depth verses time, discharge verses time or stage-discharge curve. Inflow and outflow boundaries can also be specified as open boundaries.

\section{MODEL APPLICATIONS AND RESULTS}

The model described above was applied to various illustrative problems to demonstrate applicability and validity of the model. The model was run at Courant number, $C_{n}=1.0$ for all cases. The examples considered are as described below.

The first two examples have been taken from Fennema and Choudhry ${ }^{14)}$. In the first example, bore propagation in a rectangular, horizontal and frictionless channel as a result of sudden closure of downstream gate is simulated. The second example simulates propagation of shock, resulting from sudden closure of downstream gate, in a trapezoidal channel with bottom slope and friction. The first and second examples consider the bore formed by a subcritical flow stopped suddenly by complete closure of gate. The third example demonstrates the use of proposed model in simulating a flow situation where both supercritical and subcritical flows are present. In the last example, the case of bore formation because of sudden opening of the gate has been considered. Analytical solutions for all but second example are available. Results for the above examples are discussed next.

Example 1: Shock propagation in a $5000 \mathrm{~m}$ long rectangular channel is analysed (initial flow depth, $\mathrm{h}_{0}=6.0$ $\mathrm{m}$, Initial velocity, $\mathrm{u}_{0}=3.125 \mathrm{~m} / \mathrm{s}$ ). The flow velocity at the downstream end is reduced to zero at $t=0$, thereby forming a shock or bore which propagates in the upstream direction. Results for this example were obtained at time $354+\Delta t$ seconds. Solutions obtained by Lambda ${ }^{10}$ ), Gabutti11) and MacCormack ${ }^{15)}$ schemes for this case have been taken from Fennema and Chaudhry ${ }^{14}$ ). Results obtained in the present study is compared with the results of three schemes mentioned above and analytical solution in Fig. 2 and Fig. 3.

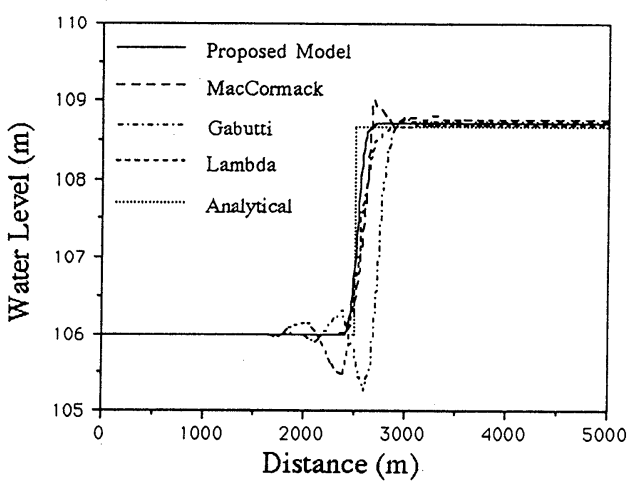

Fig.2 Example 1: Results for Rectangular Channel, No Artificial Diffusion for MacCormack, Gabutti and Lambda Schemes.

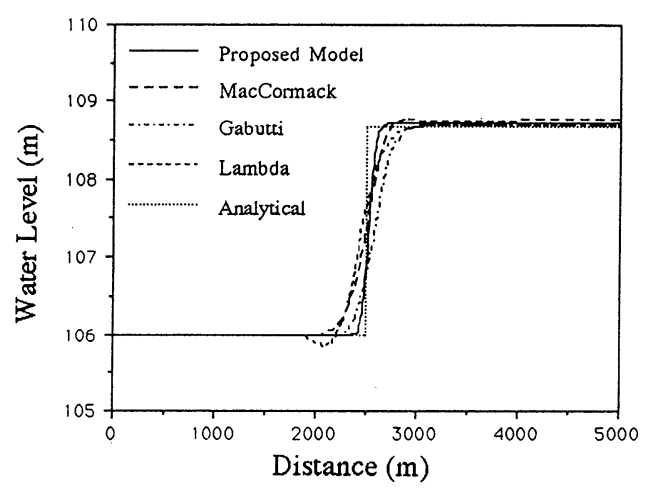

Fig.3 Example 1: Results for Rectangular Channel, Artificial Diffusion for MacCormack, Gabutti and Lambda Schemes.

As seen in Fig.2, Lambda ${ }^{10)}$, Gabutti11) and MacCormack ${ }^{15)}$ schemes oscillate near the bore when used without artificial viscosity. These three schemes are second order accurate and require some kind of artificial viscosity to damp out the oscillations near the bore. The present model does not show any oscillation near the bore and compares well with the analytical solution as well as with the previous schemes used with artificial viscosity (Fig.3). Both, the shock speed and shock height, computed by the present model are very close to the analytical solutions. 
Example 2: The channel is trapezoidal, $5000 \mathrm{~m}$ long with side slope $\mathrm{m}=1.5, \mathrm{~S}_{0}=0.0000785$, and Manning's $\mathrm{n}=0.013$. Initially, the channel has uniform flow with a velocity, $\mathrm{u}_{0}=1.47 \mathrm{~m} / \mathrm{s}$ and a flow depth $\mathrm{h}_{0}=5.79$ $m$. The flow velocity at the down stream end is reduced to zero at $t=0$ and the resulting shock or bore propagates in the upstream direction. Results using Lambda ${ }^{10)}$, Gabutti ${ }^{11)}$ and MacCormack ${ }^{15)}$ schemes for this case also have been taken from Fennema and Chaudhry ${ }^{14)}$. Results for this example were obtained at time $354+\Delta t$ seconds and are again compared with that of the three models mentioned above (Fig.4 and Fig.5). The previous schemes show oscillations near the bore (Fig.4) which have to be smoothed out by artificial diffusion (Fig.5). The present model compares well with the previous models even in this case. The analytical solution for this case is not available due to consideration of bed slope and friction.

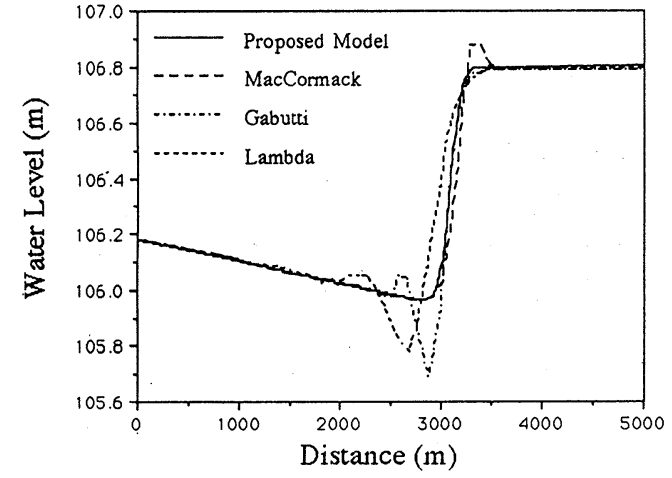

Fig.4 Example 2: Results for Trapezoidal Channel, No Artificial Diffusion for MacCormack, Gabutti and Lambda Schemes.

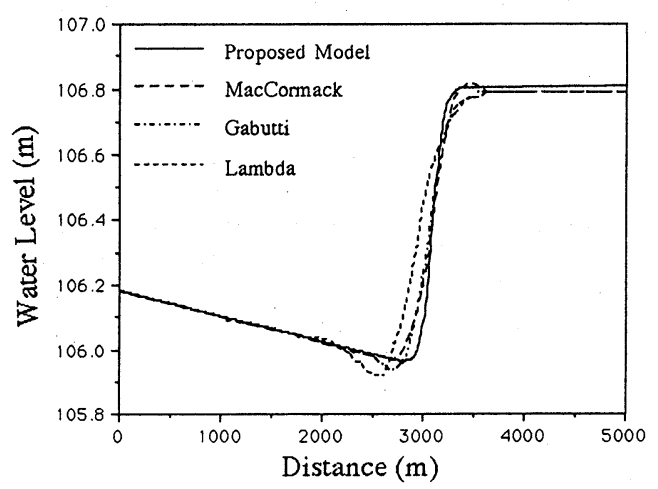

Fig.5 Example 2: Results for Trapezoidal Channel, Artificial Diffusion for MacCormack, Gabutti and Lambda Schemes.

Example 3: In this example, the initial flow in the channel of first example is replaced by flow depth, $h_{0}=6 \mathrm{~m}$ and flow velocity, $\mathrm{u}_{0}=15 \mathrm{~m} / \mathrm{s}$ giving a supercritical flow with Froude number 1.955 . The flow velocity at downstream end is reduced to zero at $t=0$. A shock is formed which travels in the upstream direction leaving a subcritical flow behind. Results for this case were obtained at time $100+\Delta t$ and $300+\Delta t$. These are plotted in Fig. 6 along with the analytical solutions. As is obvious from Fig. 6 , the present model gives excellent results for height and speed of the shock.

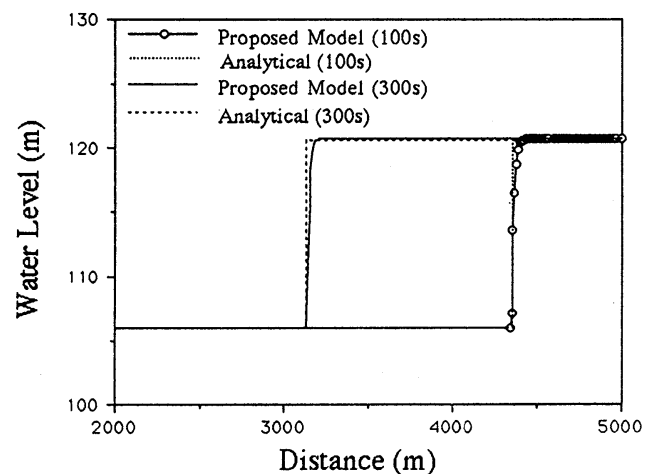

Fig.6 Example 3: Results of Supercritical Flow Reflected by Sudden Closure of Gate and Resulting Propagation of Shock.

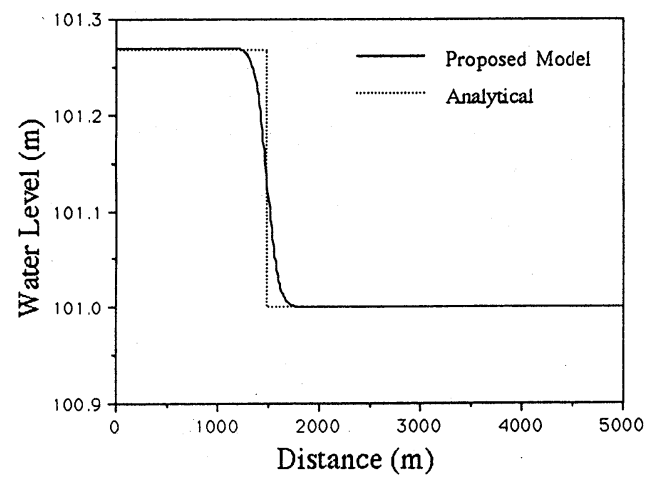

Fig.7 Example 4: Results of Shock Propagation Over Still Water after Sudden Opening of Gate 
Example 4: This example has been taken from $\mathrm{Abbott}^{3}$ ). The initial condition in the channel is described by $1 \mathrm{~m}$ deep still water throughout the channel. The downstream end is closed. A sudden and steady discharge of 1.0 $\mathrm{m}^{3} / \mathrm{s} / \mathrm{m}$ at time equal to and greater than zero is given as the upstream boundary condition. Result for this case were obtained at time $400+\Delta t$. Fig. 7 shows this result along with the analytical solution. Shock speed and height are well simulated by the present model for this case too as compared with the analytical solution.

In addition to shock heights and speeds, the steep shock front profiles are also better simulated by the proposed model when compared with previous numerical studies considered. Mass balance was tracked for all the examples. Mass balance error in the proposed model was found to be less than $1 \%$ for all the cases. It may be remarked that for the first two examples $\lambda$-scheme ${ }^{11)}$ and MacCormack ${ }^{14)}$ scheme gave a mass balance error of less than $1 \%$ but the same for Gabutti ${ }^{10)}$ scheme was about 5\% 13 ).

\section{CONCLUSIONS}

The proposed model is simple to formulate and easy to program without compromising the basic concept of directional property of signal propagation inherent in the hyperbolic partial differential equation. Besides, the boundary conditions based on method of characteristics are easily incorporated in the present model. Results of the proposed model for all the cases considered in this study are in good agreement with analytical solution as well as with solutions obtained from other models. The presented model does not have to be calibrated for any coefficient. In contrast, Lambda ${ }^{10}$ ), Gabutti ${ }^{11)}$ and MacCormack ${ }^{15}$ ) schemes require artificial diffusion ${ }^{14)}$, the coefficient of which typically varies between 0.5 and 3 . The present model, may be used with confidence to simulate such unsteady flow situations as presented in this study although the formal accuracy of the scheme is of first order.

\section{ACKNOWLEDGMENTS} research work.

The authors sincerely thank Kensetsu Gijutsu Kenkyujo Co. for their help and assistance for this

\section{REFERENCES}

1. Mahmood, K. and Yevjevich, V. (eds.), (1975), " Unsteady Flow in Open Channels", Water Resources Publications, Fort Collins, Co.

2. Cunge, J. A., Holly Jr, F. M. and Verwey, A (1980), "Practical Aspects of Computational River Hydraulics", Pitman Publishing Limited, London.

3. Abbott, M.B. (1979), "Computational Hydraulics: Elements of the Theory of Free-Surface Flows", Pitman Publishing Limited, London.

4. Chen, C. L. and Armbruster, J.T.(1980), " Dam-Break Wave Model : Formulation and Verification", J. Hyd. Div. , ASCE, Vol. 106, No. HY5, pp. 747-767.

5. Katopodes, N.D. and Strelkoff, M. (1978), "Computing Two-Dimensional Dam-Break Flood Waves", J. Hyd. Div., ASCE, Vol.104, No. HY9, pp.1269-1288.

6. Sakkas, J.G. and Strelkoff, T. (1973), "Dam-Break Flood in a Prismatic Dry Channel", J. Hyd. Div., ASCE, Vol.99, No. HY12, pp.2195-2216.

7. Sakkas J.G. and Strelkoff, T. (1976), "Dimensionless Solution of Dam-Break Flood Waves”, J. Hyd. Div., ASCE, Vol.102, No. HY2, pp. 171-184.

8. Abbott, M.B. and Ionescu, F.(1967), "On the Num erical Computation of Nearly Horizontal Flows", J. Hyd. Res., Vol.5, No. 2, pp.97-117.

9. Matsutomi, H. (1983), "Numerical Computation of Two-Dimensional Inundation of Rapidly Vartied Flows Due to Breaking of Dams", Proc. of XX Congress of the IAHR, Moscow, USSR, Sept.5-9, Vol.II, pp.479-488.

10. Moretti, G. (1979), "The $\lambda$-Scheme", Computers and Fluids, Vol.7, pp.191-205.

11. Gabutti, B. (1983), "On Two Upwind Finite-Difference Schemes for Hyperbolic Equations in NonConservation Form ", Computers and Fluids, Vol. 11, No. 3, pp.207-230.

12. Beam, R.M. and Warming, R.F. (1976), "An Implicit Finite-Difference Algorithm for Hyperbolic Systems in Conservation-Law Form ", J. Comp. Phys., Vol. 22, pp. 87-110.

13. Anderson, D.A., Tannehill, J.D. and Pletcher, R.H. (1984), "Computational Fluid Mechanics and Heat. Transfer", MacGraw-Hill, New York.

14. Fennema, R.J. and Chaudhry, M.H. (1987), "Explicit Numerical Schemes for Unsteady Free-Surface Flows With Shocks", Water Res. Res., Vol.22, No.13, pp. 1923-1930.

15. MacCormack, R. W. (1971), "Numerical Solution of the Interaction of a Shock Wave with a Laminar Boundary Layer", Lecture notes in Physics, Vol. 8, pp. 151-163, Springer - Verlag, New York. 